\title{
Astronomical databases of Nikolaev Observatory
}

\author{
Y. Protsyuk and A. Mazhaev \\ Nikolaev Astronomical Observatory, Ukraine \\ email: yuri@mao.nikolaev.ua
}

\begin{abstract}
Several astronomical databases were created at Nikolaev Observatory during the last years. The databases are built by using MySQL search engine and PHP scripts. They are available on NAO web-site http://www.mao.nikolaev.ua.
\end{abstract}

Keywords. astronomical data bases: miscellaneous, catalogs

\section{Development of the astronomical databank}

Development of the astronomical databank of NAO has begun since 1995, the year of the first CCD observations with the Axial Meridian Circle. Some astrometric catalogues and data of the CCD observations were included into this databank in 1995 (Kovalchuk et al. 1997).

A local area network has considerably grown since 1995, and today it includes about 50 PC in four separate buildings. Two dedicated servers manage the network. Control computers of three CCD telescopes, such as the Axial Meridian Circle (AMC), the Multi Channel Telescope (MCT), the Fast Robotic Telescope (FRT) are also connected to the network.

All obtained data and results of data processing are added to the common databank of NAO (Protsyuk et al. 2005, Pinigin et al. 2005). The daily average volume of the new astronomical information obtained from the CCD instruments makes from 300MB up to $7 \mathrm{~GB}$, depending on the purposes and conditions of observations. The total volume of information was about 340GB in the middle of 2007 (Fig. 1). The total volume of information obtained from other sources such as astronomical catalogues makes about 50GB.

The data of CCD observations obtained from the telescopes of NAO and other observatories (Fig. 2) has been handled in automatic and semi-automatic mode for determination of coordinates of observed stars, minor planets and other objects since 1996 (Kovalchuk et al. 1997, Protsyuk 2000).

All received data are stored on servers in two copies, namely, working and backup. Also we make two copies of observational data as archives on DVD. One copy is available for users. Another copy is stored in a central storage of NAO. All raw observational data have been stored in FITS format since 1998. Also, the databank of NAO includes the results of data processing as pixel coordinates for all star-shaped objects captured on the CCD frames.

The network has been connected to the Internet via the first dedicated line since the end of 2004 and the second one since the middle of 2007 (Fig. 3). It allows us to carry out fast transmission of raw observational data through the Internet.

The glass library of NAO contains more than 8000 photographic plates. We have started a program of digitizing of photographic plates with resolution of 2400DPI for 


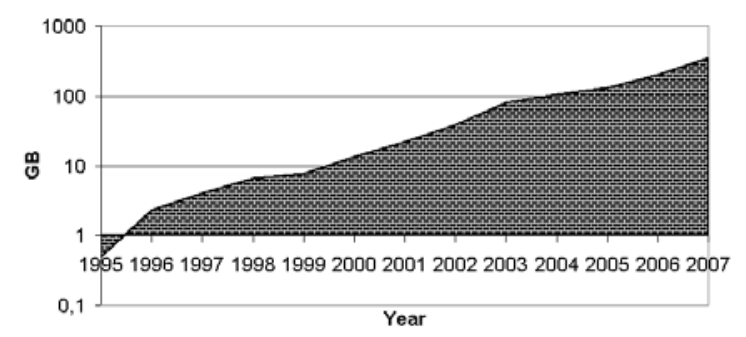

Figure 1. Growth of data volume obtained during CCD observations, GB

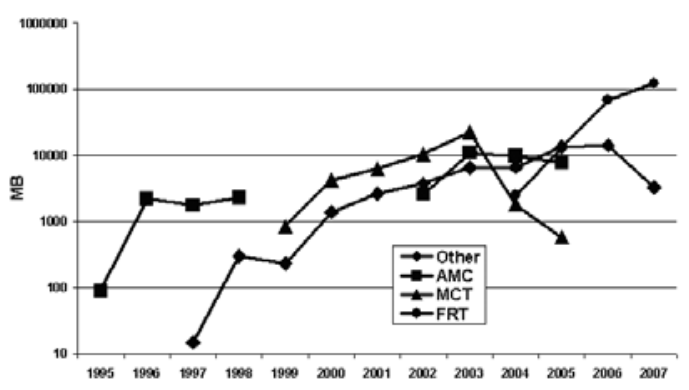

Figure 2. Volume of data obtained from different telescopes, MB

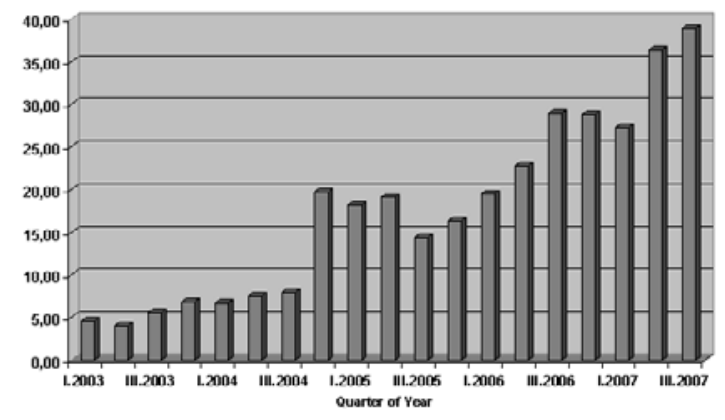

Figure 3. Volume of the Internet traffic, GB

astrometry and 600DPI for preview since the beginning of 2007 (Fig. 4). Now we have about 1000 images for preview and 150 for astrometry.

We have also started digitizing and optical character recognition of printed catalogues and scientific publications (Fig. 5). 27 stellar catalogues were digitized and included in the database of catalogues.

\section{Development of the astronomical databases}

Development of astronomical databases has started since 2004. Several astronomical databases were created during last years. They are available on NAO web site $h t t p$ : //www.mao.nikolaev.ua (Fig. 7):

- The databases of photographic and CCD observations;

- The database of astrometric catalogues;

- The catalogue of artificial satellites and space debris;

- The database of ionosphere sounding. 


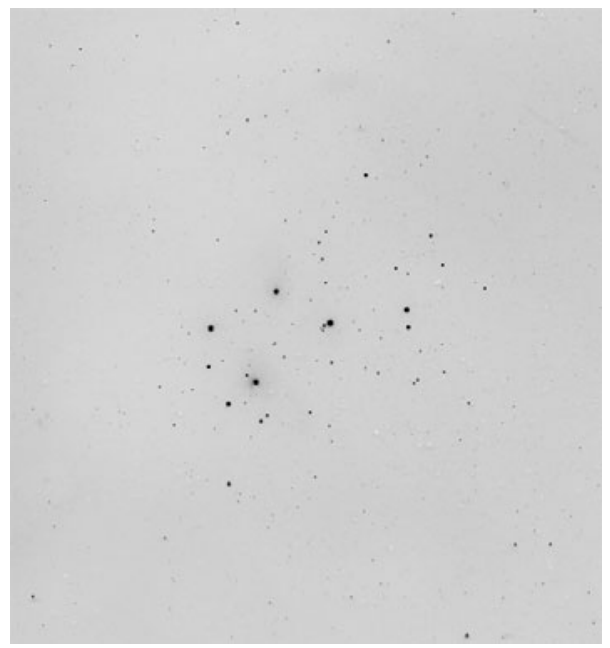

Figure 4. Sample of digitized photoplate

\begin{tabular}{|c|c|c|c|c|c|c|}
\hline & & & & & & \\
\hline 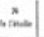 & $\stackrel{*}{*}$ & 4 & & $x-1$ & $n$ & $x-x_{0}^{n}$ \\
\hline ; & : & 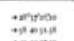 & & & 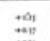 & 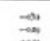 \\
\hline & 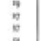 & 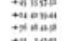 & 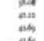 & 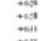 & 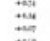 & \\
\hline & 湿 & 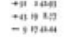 & in & 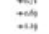 & $\approx$ & to \\
\hline & 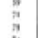 & Fin & 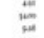 & $\approx$ & $\approx$ & $\Rightarrow$ \\
\hline & $\vdots$ & 饬 & 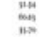 & 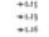 & : & \pm \\
\hline & $\vdots$ & Fine & 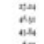 & $\Xi$ & 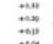 & $\underline{z}$ \\
\hline & 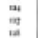 & 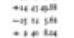 & 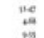 & : & : & $\exists$ \\
\hline & 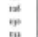 & t) & it & $\Rightarrow$ & tivis & $\exists$ \\
\hline & 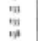 & 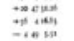 & 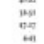 & : & 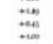 & 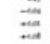 \\
\hline & 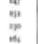 & 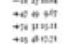 & 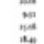 & $\Xi$ & 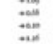 & 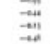 \\
\hline & $z$ & 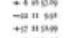 & 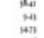 & $\Xi$ & 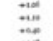 & \pm \\
\hline 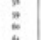 & 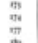 & 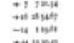 & $=$ & 落 & : & $\exists$ \\
\hline 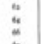 & 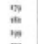 & thes & 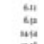 & $\vec{t}$ & : & 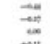 \\
\hline $\begin{array}{l}n \\
n \\
n\end{array}$ & $=$ & $\begin{array}{l}7120 \\
078\end{array}$ & 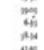 & 茫 & 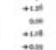 & $\Rightarrow$ \\
\hline & 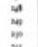 & 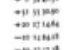 & 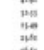 & \pm & : & $\exists$ \\
\hline & & ton & & $+\infty$ & & \\
\hline
\end{tabular}

Figure 5. Sample of digitized catalogue

Overall size of databases is about 200MB. Development of new databases is continuing. The databases allow users to search information about observations by using seven different graphical interfaces. The databases are built by using MySQL search engine and PHP scripts.

Now these databases contain information about CCD observations obtained in 1996 2006 and photographic observations obtained in $1929-1931$ and $1961-1999$ (Fig.6). The database of catalogues can be connected to interactive client side application such as Aladin allowing the user to visualize data in the field of search. The catalogues can also be visualized in the VOTable or ASCII formats by using such application as TopCat, which allows the user to carry out wide range of data processing.

The databases of photographic and CCD observations allow users to search information by using five different graphical interfaces. The search is possible by coordinates or by 


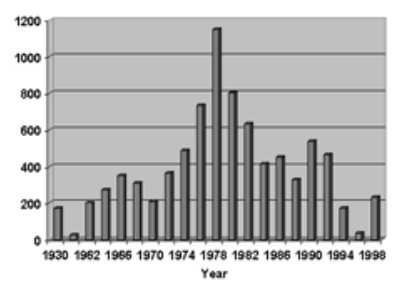

$\mathrm{a}$

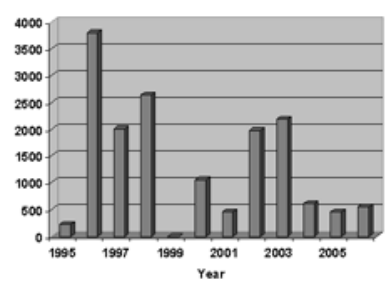

d

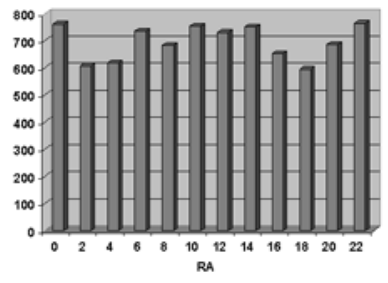

$\mathrm{b}$

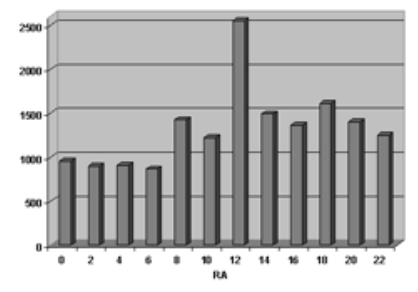

e

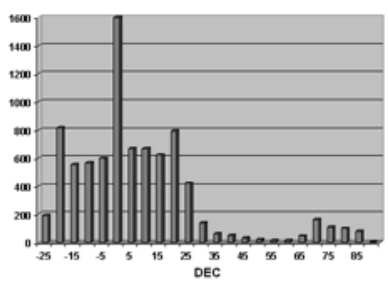

C

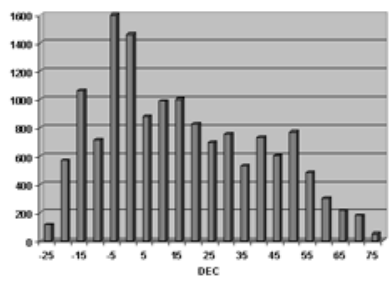

f

Figure 6. Distribution of photoplates (top) and CCD frames (bottom) by year of observation $(\mathrm{a}, \mathrm{d}), \mathrm{RA}(\mathrm{b}, \mathrm{e})$ and DEC (c,f) in databases of photographic and CCD observations.

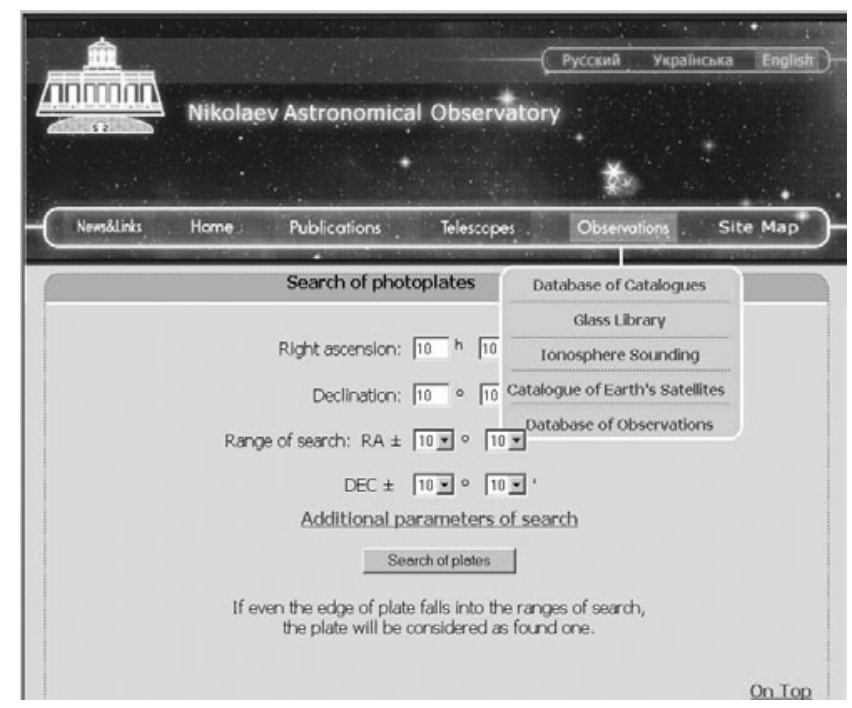

Figure 7. View of search page on web site

object. The databases of photographic and CCD observations allow users to search plates by using the center of search and ranges of search in right ascension and declination. The database of photographic observations allows users to search plates by using the period of observations and observational campaign. The database of CCD observations allows users to search CCD images by using the period and objects of observations. The database of observations also allows users to search plates and CCD frames simultaneously.

The database of astrometric catalogues allows users to search information by using the coordinate center and radius of search.

Database of ionosphere sounding has been recorded since 2002. The graphical data is updated every 5 minutes and is available for online search (Fig. 8).

The Earth satellites in catalogue are separated in two groups: Geostationary Earth Orbit (GEO) satellites and space debris; Low Earth Orbit (LEO) satellites. GEO 


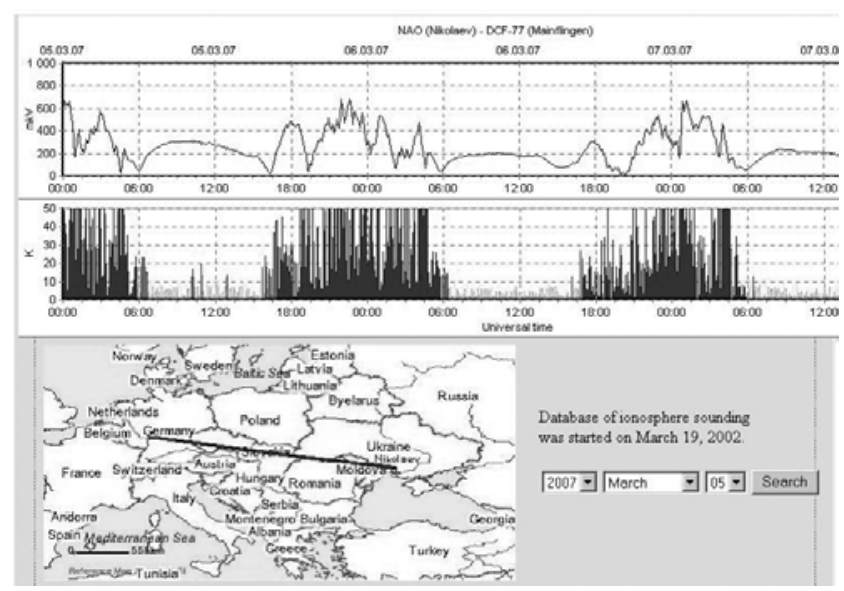

Figure 8. View of ionosphere sounding record

satellites and space debris were observed with the Multi-Channel Telescope in 2002-2004 and with the Fast Robotic Telescope in 2004 - 2007. LEO satellites were observed with the Fast Robotic Telescope in $2004-2007$.

Several our catalogues were included into the international databases, such as the CDS in Strasbourg, the IMCCE in Paris, the WFPDB in Sofia. The database of astrometric catalogues was recorded in the registry of the NVO in the USA. We hope, that in the near future all our databases will be integrated to the registries of the International Virtual Observatory.

\section{References}

Kovalchuk, A. N., Pinigin, G. I., Protsyuk, Yu. I., \& Shulga, A. V. 1997, Journees 1997 Systemes de Reference Spatio-Temporels. Prague., 14-17

Protsyuk, Yu. 2000, Baltic Astronomy, 9, 554-556

Protsyuk, Yu., Pinigin, G., \& Shulga, A. 2005, Kinematics and Physics of Celestial Bodies, Suppl. Ser., 5, 580-584

Pinigin, G., Protsyuk, Yu., \& Shulga, A. 2005, Romanian Astronomical Journal, Suppl. , 15, $51-56$ 